\title{
miR-181b inhibits chemoresistance in cisplatin-resistant H446 small cell lung cancer cells by targeting Bcl-2
}

\author{
Hui-Ning Liu, Peng Qie, Guang Yang, Yong-Bin Song
}

Department of Thoracic Surgery, Hebei General Hospital, Shijia Zhuang, China

Submitted: 12 July 2017

Accepted: 22 August 2017

Arch Med Sci 2018; 14, 4: 745-751

DOI: https://doi.org/10.5114/aoms.2018.73131

Copyright @ 2017 Termedia \& Banach

\section{Abstract}

Introduction: MicroRNAs (miRNAs) are a group of small non-coding RNAs that affect multiple aspects of tumor biology including chemo resistance. miR-181b has been reported to modulate multidrug resistance in non-small cell lung cancer cells. This study was undertaken to determine the role of miR-181b in chemo resistance of small cell lung cancer cells.

Material and methods: This study was undertaken to determine the role of miR-181 b in chemoresistance of small cell lung cancer cells with use of qRt-PCR, WB, bioinformatics analysis, and double luciferase reporter system.

Results: Our data showed that miR-181b was significantly downregulated in cisplatin-resistant $\mathrm{H} 446$ small cell lung cancer cells, compared to parental cells, compared to parental cells. Ectopic expression of miR-181b inhibited cell proliferation and invasion in cisplatin-resistant $\mathrm{H} 446$ cells $(p=0.023)$. Moreover, overexpression of miR-181b increased the susceptibility of cisplatin-resistant $\mathrm{H} 446$ cells to cisplatin. Mechanistic investigations demonstrated that miR-181b inhibited B-cell lymphoma-2 ( $\mathrm{Bcl}-2)$ expression by binding to the 3'-untranslated region. Overexpression of Bcl-2 reversed miR-181b-mediated chemo sensitization, which is accompanied by a reduced apoptotic response. Conclusions: Taken together, this work demonstrated that miR-181b might have the ability to overcome chemo resistance of small cell lung cancer cells, and restoration of this miRNA may represent a potential therapeutic strategy for improving chemo sensitivity in small cell lung cancer.

Key words: miR-181b, small cell lung cancer cells, Bcl-2, cisplatin-resistant.

\section{Introduction}

Small-cell carcinoma (also known as small-cell lung cancer, or oat-cell carcinoma) is a type of highly malignant cancer that most commonly arises within the lung, although it can occasionally arise in other body sites, such as the cervix, prostate, and gastrointestinal tract [1-4]. Compared to non-small cell carcinoma, small cell carcinoma has a shorter doubling time, higher growth fraction, and earlier development of metastases. Although advances in the diagnosis of small-cell lung cancer patients in early stages have been achieved and efficient treatments have been developed, the prognosis of patients with small-cell lung cancer remains poor, and their 5 -year survival rate is less than $50 \%$ [5]. The exact mechanisms underlying small-cell lung cancer formation and progression are also unknown. Thus, further investigations on the molecular mechanism of small-cell lung cancer occurrence and development may be advantageous to identify novel biomarkers and therapeutic strategies to improve the survival of patients with this disease.

\author{
Corresponding author: \\ Hui-Ning Liu \\ Department of \\ Thoracic Surgery \\ Hebei General Hospital \\ 348 He Peng Road \\ Shijiazhuang 050051, China \\ Phone: 86-311-85989926 \\ Fax: 86-311-85989926 \\ E-mail: hbhx_thn@sina.com
}


MicroRNAs (miRNAs) are a subtype of endogenous, single strand, noncoding and short RNAs 1925 nucleotides in length [6-9]. miRNAs negatively modulate the expression of protein-coding genes through binding to complementary sequences in the 3'-untranslated regions (3'-UTRs) of their target genes, resulting in either mRNA cleavage and/or translational repression. miRNAs have been proven to play crucial roles in a variety of critical cellular processes, including cell proliferation, apoptosis, differentiation, invasion, angiogenesis and metastasis [10]. Recently, increasing numbers of studies have reported that numerous miRNAs are aberrantly expressed and involved in the occurrence and development of human cancer [11-13]. In small-cell carcinoma, several abnormally expressed miRNAs and their biological roles have been reported. For instance, miR-422a was downregulated in OS tissues and cell lines. Upregulation of miR-422a suppressed small-cell carcinoma cell proliferation and invasion, and improved paclitaxel and cisplatin-mediated apoptosis [14].

miR-181b is abnormally expressed in several types of human cancer [15-19]. This finding suggests that miR-181b may play important roles in these types of cancer. However, the detailed expression level, biological roles and underlying mechanism of miR-181b in cisplatin-mediated apoptosis remain unknown. Therefore, it is essential to investigate the expression level and the role of miR-181b in chemo resistance of small cell lung cancer cells and its underlying molecular mechanism, which may a provide novel and efficient therapeutic strategy for patients with small cell lung cancer.

\section{Material and methods}

\section{Cell lines and transfection}

Cisplatin-resistant cell line H446 and the corresponding parental cells were purchased from the Cell Bank of Type Culture Collection of the Chinese Academy of Sciences (Shanghai, China) and cultured in Dulbecco's Modified Eagle Medium (Gibco; Thermo Fisher Scientific, Inc., Waltham, MA, USA) supplemented with $10 \%$ fetal bovine serum (FBS), 100 units $/ \mathrm{ml}$ penicillin and $100 \mathrm{mg} / \mathrm{ml}$ streptomycin (all from Gibco; Thermo Fisher Scientific, Inc., Waltham, MA, USA). All of the cells were grown at $37^{\circ} \mathrm{C}$ in a humidified environment with $5 \% \mathrm{CO}_{2}$.

miR-181b mimics and negative control miRNAs mimics (miR-NC) were synthesized by Shanghai GenePharma Co., Ltd. (Shanghai, China). The Bcl-2-expressing vector (pcDNA3.1- Bcl-2) and empty vector (pcDNA Bcl-2) were obtained from Ribobio (Guangzhou, China). The cells were seeded in a six-well plate (Corning, Inc., Corning, NY, USA) at a density of $60-70 \%$ confluence, incubated overnight at $37^{\circ} \mathrm{C}$ and transfected with $\mathrm{Li}$ - pofectamine 2000 (Invitrogen Life Technologies, Carlsbad, CA, USA) according to the manufacturer's instructions.

\section{Reverse transcription-quantitative polymerase chain reaction (RT-qPCR)}

RNA extraction was performed using a Trizol reagent (Invitrogen, Carlsbad, CA, USA) according to the manufacturer's instructions. miRNA was reverse-transcribed using a TaqMan MicroRNA Reverse Transcription Kit (Applied Biosystems, Foster City, CA, USA) and qPCR was conducted with a TaqMan MicroRNA PCR Kit (Applied Biosystems, Foster City, CA, USA), according to the manufacturer's instructions. To quantity Bcl-2 mRNA expression, reverse transcription was performed with the M-MLV Reverse Transcription system (Promega Corporation, Madison, WI, USA). SYBR Premix Ex Taq (TaKaRa, Dalian, China) was used to measure Bcl-2 mRNA expression. All RT-qPCR was performed using the ABI7500 Real-time PCR system (Applied Biosystems, Carlsbad, CA, USA). The endogenous U6 small nuclear RNA (U6 snRNA) and $\beta$-actin were amplified as internal controls for miR-181b and Bcl-2 mRNA, respectively. Relative miRNA or mRNA expression was determined using the $2^{-\Delta \Lambda c t}$ method.

\section{Cell counting Kit-8 (CCK8) assay}

The CCK8 assay (Dojindo Molecular Technologies, Inc., Kumamoto, Japan) was used for analyzing the cell proliferation following the manufacturer's instructions. Briefly, transfected cells were collected at $24 \mathrm{~h}$ after transfection, and reseeded into 96 -well plates at a density of $3 \times 10^{3}$ cells per well. Cells were incubated at $37^{\circ} \mathrm{C}$ and cell proliferation was examined every $24 \mathrm{~h}$ according to the manufacturer's instructions (0 h, 24 h, 48 h, 72 h). Ten $\mu$ l of CCK8 solution was added into each group of cells and incubated at $37^{\circ} \mathrm{C}$ for $4 \mathrm{~h}$. The absorbance of each sample was determined at a wavelength of $450 \mathrm{~nm}$ using an automatic multi-well spectrophotometer (Bio-Rad Laboratories, Inc., Hercules, CA, USA). All samples were analyzed in triplicate and repeated three times.

\section{Cell invasion assay}

Cell invasion assays were conducted using Transwell chamber inserts (Costar; Corning Life Sciences, Cambridge, MA, USA) with Matrigel (BD Biosciences, San Jose, CA, USA) according to the manufacturer's protocol. Briefly, transfected cells were collected at $48 \mathrm{~h}$ after transfection, and seeded into the upper chamber of the insert at a density $5 \times 10^{4}$ in $200 \mu \mathrm{l}$ of FBS-free medium. The bottom of the insert was filled with DMEM containing $20 \%$ FBS to serve as a chemo attractant. After $20 \mathrm{~h}$ of incubation, the cells remaining 
on the upper membrane were carefully removed using cotton swabs. The invasive cells were fixed in $100 \%$ methanol, stained with $0.5 \%$ crystal violet, washed with PBS, and dried in air. Photographs of five manually selected fields of the invasive cells were taken at 200x magnification and counted under an inverted light microscope (Olympus, Tokyo, Japan).

\section{miR-181b targets prediction and luciferase reporter assay}

The target genes of miR-181b were predicted using two miRNA targeted-gene databases: TargetScan (http://www.targetscan.org/index. html) and Pictar (http://pictar.mdc-berlin.de/). HEK 293T cells (ATCC) were seeded into 24-well plates at a density of $40-50 \%$ confluence. The next day, HEK 293T cells were transfected with pmirGLOBcl-2-3'UTR-wild type (Wt) or pmirGLO-Bcl-23'UTR-mutant (Mut) (GenePharma), and miR-181b mimics or miR-NC using Lipofectamine 2000. Cells were then incubated at $37^{\circ} \mathrm{C}$ in $5 \% \mathrm{CO}_{2}$ for 2 days. Transfected cells were harvested and subjected to the luciferase reporter assay using the Dual-Luciferase Reporter assay system (Promega Corporation, Madison, WI, USA). Renilla luciferase was used as an internal control to correct for differences in both transfection and harvesting efficiencies.

\section{Statistical analysis}

All of the data were represented as mean \pm S.D. or box plots. Data were analyzed using SPSS 18.0 software (SPSS, Inc, Chicago, IL). Differences between groups were compared with Student's t-test or one-way ANOVA plus multiple comparisons. The association between miR-181b and $\mathrm{Bcl}-2$ mRNA expression was analyzed by Spearman's correlation analysis. A double-tailed $p$-value less than 0.05 was considered to be statistically significant.

\section{Results}

miR-181b was downregulated in cisplatinresistant H446 small cell lung cancer cells

RT-qPCR was used to examine miR-181b expression in cisplatin-resistant $\mathrm{H} 446$ small cell lung cancer cells and associated parental cells. The data showed that the miR-181b expression level was reduced in cisplatin-resistant $\mathrm{H} 446$ in comparison with that in parental cells (Figure $1, p<0.05$ ).

miR-181b overexpression inhibits cell proliferation and invasion in cisplatinresistant $\mathrm{H} 446$

To explore the roles of miR-181b in cisplatin-resistant cells, $\mathrm{H} 446$ was transfected with miR-181b mimics to increase the endogenous miR-181b expression. Using RT-qPCR, we found that miR-181b was markedly upregulated in cisplatin-resistant cells, H446 transfected with the miR-181b mimics compared with the cells transfected with miR-NC (Figure $2 \mathrm{~A}, p<0.05)$. To investigate the effect of miR-181b on cisplatin-resistant cell proliferation, the CCK8 assay was performed in $\mathrm{H} 446$ cells transfected with miR-181b mimics or miR-NC. The miR-181b upregulation inhibited the proliferation of cisplatin-resistant cells (Figure 2 B). A cell invasion assay was conducted to evaluate the effects of miR-181b on the invasion capacity of cisplatin-resistant cells. The restored expression of miR-181b reduced the invasive capacities of cisplatin-resistant cells $(p<0.05)$ (Figure 2 C). These observations revealed that miR-181b functioned as a tumor suppressor in cisplatin-resistant cells.

\section{$\mathrm{Bcl}-2$ is a direct target of miR-181b}

Bioinformatics analysis was conducted to predict the putative targets of miR-181b and to investigate the molecular mechanism by which miR-181b repressed cisplatin-resistant cell proliferation and invasion. Numerous genes were identified as potential targets of miR-181b, and Bcl-2 (Figure $3 \mathrm{~A}$ ) was selected for further confirmation. A luciferase reporter assay was carried out to investigate whether $\mathrm{Bcl}-2$ was a target for miR-181b. HEK293T cells were co-transfected with pGL3-Bcl2-3'-UTR Wt or pGL3Bcl-2-3'-UTR Mut and miR$181 \mathrm{~b}$ mimics or miR-NC. We found that the upregulation of miR-181b significantly reduced the luciferase activity of pGL3-Bcl-2-3'-UTR Wt (Figure $3 \mathrm{~B}, p<0.05)$. By contrast, the cells transfected with pGL3-BCl-21-3'-UTR Mut were unaffected. This finding suggested that miR-181b could directly target the $3^{\prime}$-UTR of $\mathrm{BCl}-2$. To confirm the endogenous regulatory role of $\mathrm{miR}-181 \mathrm{~b}$ in relation to $\mathrm{BCl}-2$, we determined the $\mathrm{BCl}-2$ expression in cisplatin-resistant cells, H446 cells transfected

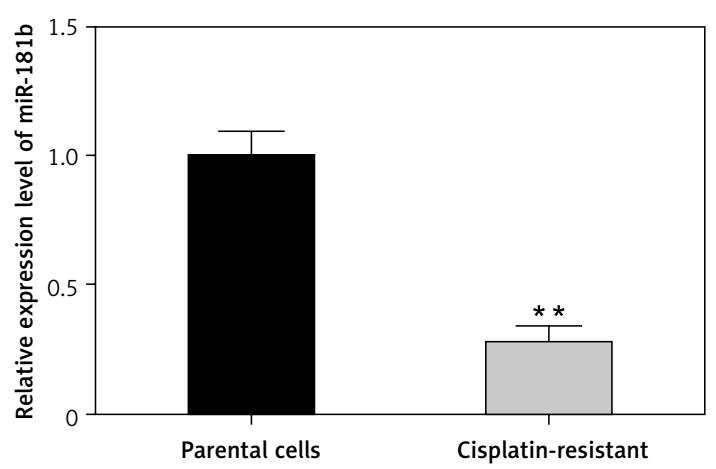

Figure 1. miR-181b expression was downregulated in cisplatin-resistant $\mathrm{H} 446$ cells RT-qPCR analysis of miR-181b expression in cisplatin-resistant $\mathrm{H} 446$ cells and nornal $\mathrm{H} 446$ cells 
A

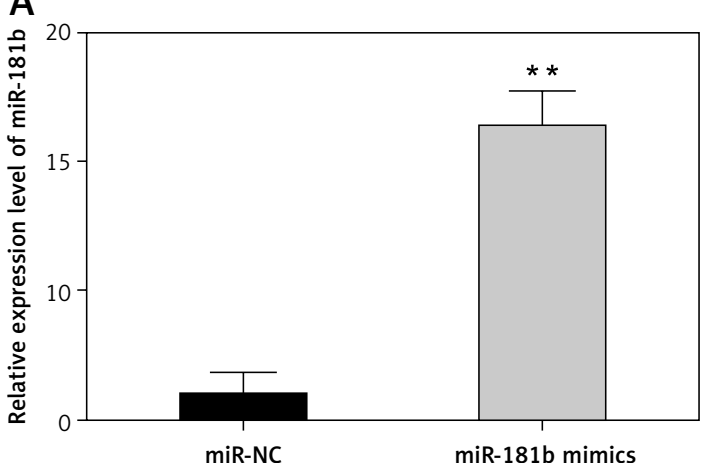

C
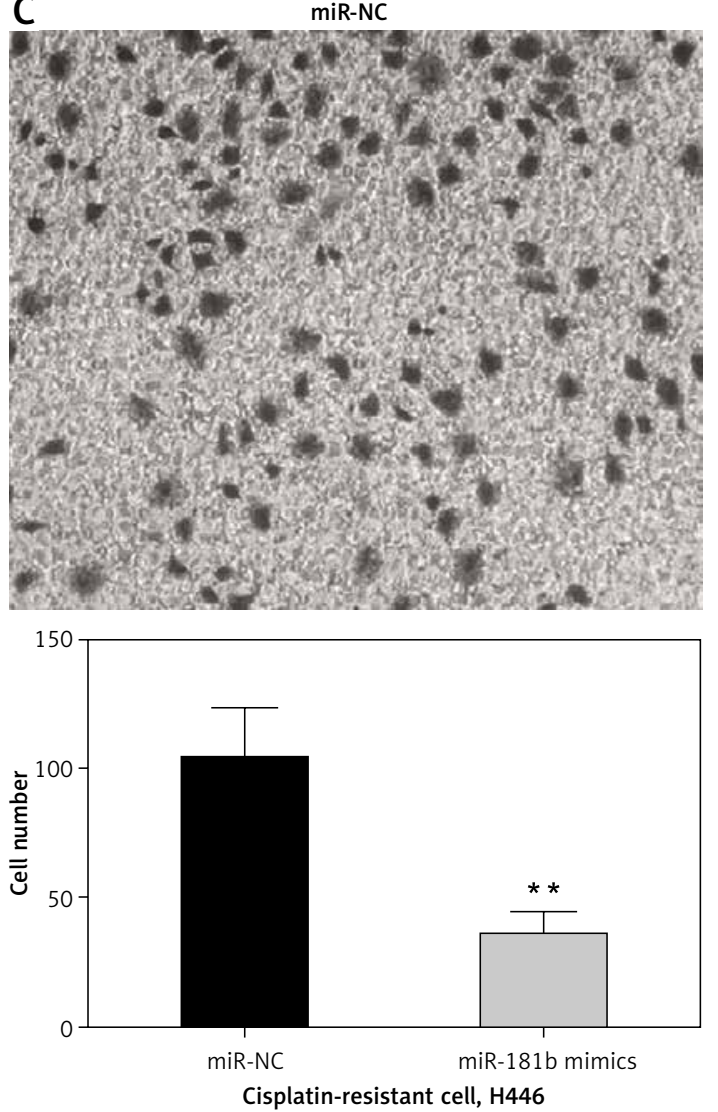

with miR-181b mimic or miR-NC. The mRNA levels of $\mathrm{Bcl}-2$ were downregulated in cisplatin-resistant cells transfected with miR-181b mimic $(p<0.05)$ (Figure $3 \mathrm{C}$ ). These results suggested that $\mathrm{BCl}-2$ might be a direct target of miR-181b in cisplatinresistant $\mathrm{H} 446$ cells.

\section{Discussion}

Dysregulation of miRNAs is a common event in many human tumors, and dysregulated miRNAs may play important roles in tumorigenesis and tumor development by functioning as tumor suppressors or oncogenes. Moreover, accumulated studies have demonstrated that targeting miRNA
B

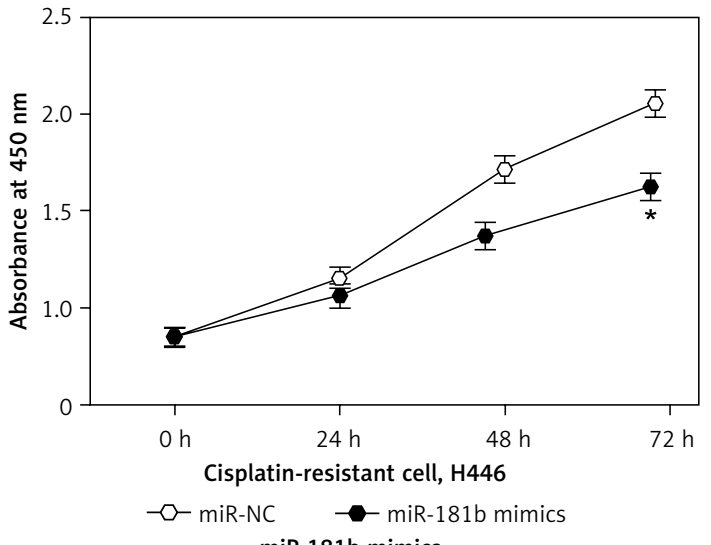

miR-181b mimics

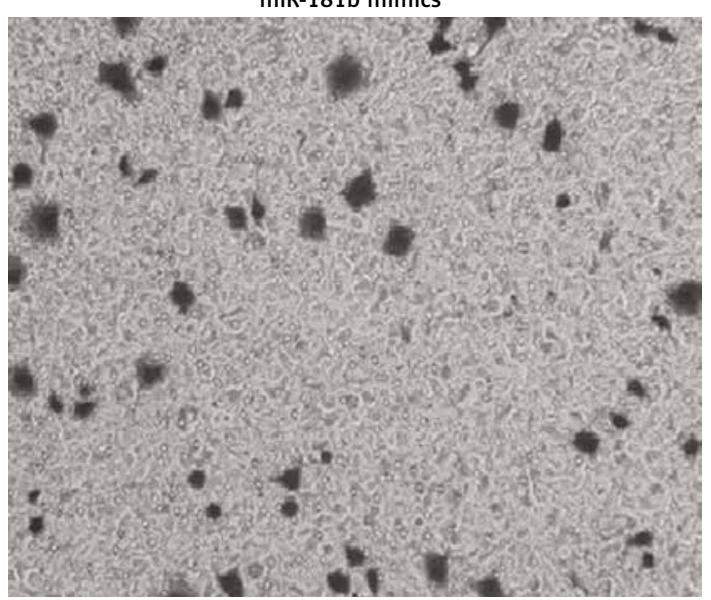

Figure 2. miR-181b overexpression inhibited proliferation and invasion of cisplatin-resistant $\mathrm{H} 446$ cells. A - Transfection efficiency of miR-181b mimic in cisplatin-resistant $\mathrm{H} 446$ cells, as revealed by RT-qPCR $48 \mathrm{~h}$ after transfection with miR-NC as a negative control. B - CCK8 assay was performed to examine the effects of miR-181b on proliferation of cisplatin-resistant H446 cells. Compared with the proliferation of cells transfected with miR-NC, the proliferation of cisplatin-resistant $\mathrm{H} 446$ cells transfected with miR-181b mimic was reduced. C - Cell invasion assay of cisplatin-resistant H446 cells transfected with miR-181b mimic or miR-NC

using chemically modified oligonucleotides is capable of invalidating the functions of miRNAs in human cancer, which may provide the theoretical basis for miRNA-based targeted therapy in specific cancer. Hence, investigation of miRNA expression patterns and biological roles, as well as their underlying molecular mechanisms, may be developed as a valuable strategy for cancer treatment. In this study, we found significant downregulation of miR-181b in cisplatin-resistant $\mathrm{H} 446$ cells. Furthermore, functional experiments revealed that enforced expression of miR-181b suppressed cisplatin-resistant $\mathrm{H} 446$ cell proliferation and invasion. Moreover, $\mathrm{Bcl}-2$ was demonstrated as a direct and functional target of miR-181b in 
A

BCl-2-3'-UTR Wt 5'...UCUGUAUUAACUUUGGAAUGUAC hsa-miR-181b 3' UGGGUGGCUGUCGUUACUUACAA

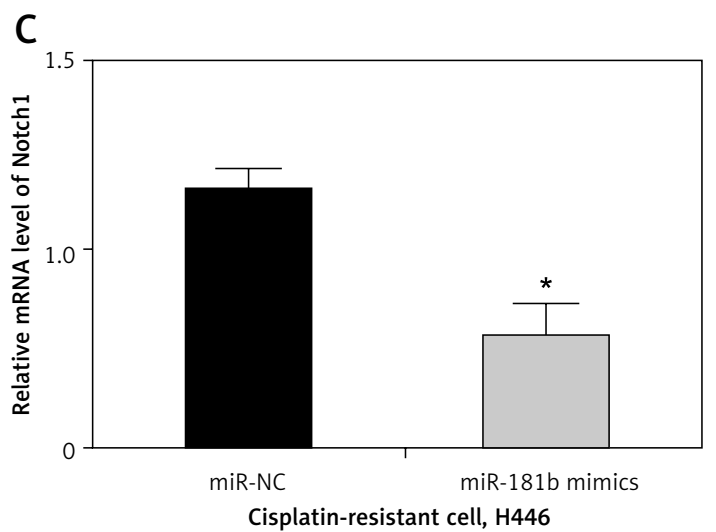

B

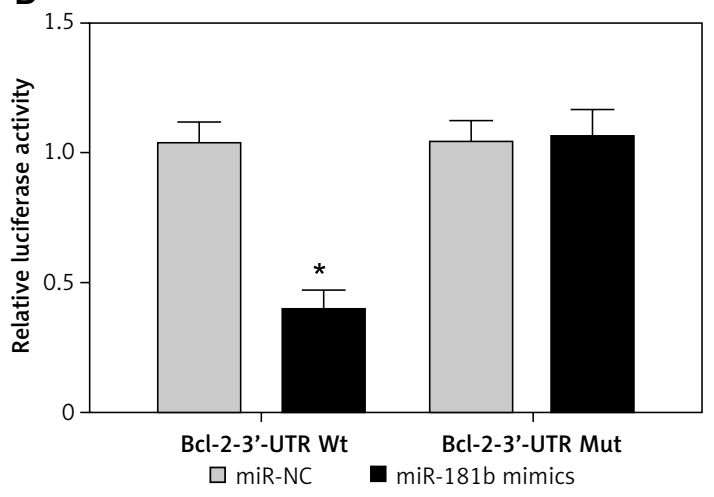

Figure 3. Bcl-2 is a direct target gene of miR-181b. A - Putative binding sites of miR-181b in the $3^{\prime}-$ UTR of Bcl-2. B - Relative luciferase activity was detected in HEK293T cells co-transfected with Wt or Mut vector and miR-181 mimic or miR-NC after 48 h. C - mRNA levels of Bcl-2 in cisplatin-resistant H446 cells transfected with miR-181b mimics or miR-NC were determined through RT-GPCR

cisplatin-resistant $\mathrm{H} 446$ cells. These findings may be the first time that the expression and roles of miR-181b in cisplatin-resistant H446 cells have been revealed.

Aberrant miR-181b expression has been observed in various human cancers [20-23]. For instance, miR-181b is weakly expressed in hepatocellular carcinoma. miR-181b expression is correlated with multiple tumor nodes, high Edmondson-Steiner grading, advanced tumor node metastasis stage and venous infiltration of hepatocellular carcinoma patients. miR-181b is also validated as a novel prognostic indicator to predict the 5-year overall and disease-free survival of hepatocellular carcinoma patients. In gastric cancer, the miR-181b expression level is downregulated in tumor tissues and related to advanced clinical stage for patients with gastric cancer. miR-181b downregulation is also observed in nonsmall cell lung cancer and colorectal cancer. However, miR$181 \mathrm{~b}$ expression is increased in oral squamous cancer tissues and cell lines. These findings suggested that miR-181b expression exhibits tissue specificity and may be a diagnostic and prognostic marker for cancers. These findings also suggested that miR-181b is involved in the tumorigenesis and progression of these cancer types and is a promising therapeutic target for the treatment of cancers.

miRNAs perform their biological roles by negatively regulating their target genes. Therefore, the identification and characterization of the targets of altered miRNAs may help elucidate the molecular mechanisms involved in carcinogenesis and progression [15, 16, 24-30]. In this study, $\mathrm{Bcl}-2$ was validated as a novel direct target of miR-181b in small cell lung cancer. TargetScan and Pictar predicted that $\mathrm{Bcl}-2$ was a potential miR-181b target. Secondly, the luciferase reporter assay revealed that miR-181b could directly target the 3'-UTR of Bcl-2. Thirdly, RT-qPCR analysis revealed that miR-181b reduced $\mathrm{Bcl}-2$ expression at the mRNA level. These findings indicated that miR-181b was involved in cisplatin-resistant small cell lung cancer carcinogenesis and progression by directly targeting $\mathrm{Bcl}-2$.

$\mathrm{Bcl}-2$ is localized to the outer membrane of mitochondria, where it plays an important role in promoting cellular survival and inhibiting the actions of pro-apoptotic proteins [31, 32]. The pro-apoptotic proteins in the $\mathrm{Bcl}-2$ family, including Bax and Bak, normally act on the mitochondrial membrane to promote permeabilization and release of cytochrome $\mathrm{C}$ and reactive oxygen species, which are important signals in the apoptosis cascade. These pro-apoptotic proteins are in turn activated by $\mathrm{BH} 3$-only proteins, and are inhibited by the function of $\mathrm{BCl}-2$ and its relative $\mathrm{BCl}-\mathrm{Xl}$. Cancer can be seen as a disturbance in the homeostatic balance between cell growth and cell death. Over-expression of anti-apoptotic genes, and under-expression of pro-apoptotic genes, can result in the lack of cell death that is characteristic of cancer. An example can be seen in lymphomas [33]. The over-expression of the anti-apoptotic $\mathrm{Bcl}-2$ protein in lymphocytes alone does not cause cancer. But simultaneous over-expression of $\mathrm{Bcl}-2$ and the proto-oncogene myc may produce aggressive B-cell malignancies including lymphoma. In follicular lymphoma, a chromosomal translocation commonly occurs between the fourteenth and the eighteenth chromosomes which places the $\mathrm{Bcl}-2$ gene from chromosome 18 next to the immunoglobulin heavy chain locus on chro- 
mosome 14. This fusion gene is deregulated, leading to the transcription of excessively high levels of $\mathrm{Bcl}-2$. This decreases the propensity of these cells for apoptosis [34].

In conclusion, miR-181b was downregulated in cisplatin-resistant small cell lung cancer. miR$181 \mathrm{~b}$ inhibited cisplatin-resistant small cell lung cancer cell proliferation and invasion by directly targeting $\mathrm{Bcl}-2$. These results suggested that miR-181b might have the ability to overcome chemoresistance of small cell lung cancer cells, and restoration of this miRNA could be a potential therapeutic strategy for improving chemosensitivity in small cell lung cancer.

\section{Conflict of interest}

The authors declare no conflict of interest.

\section{References}

1. Shimasaki $\mathrm{N}$, Inoue $\mathrm{K}$, Nishigawa $\mathrm{H}$, et al. Combined small cell carcinoma and sarcomatoid squamous cell carcinoma in the renal pelvis. Int J Urol 2005; 23: 686-9.

2. Chang CY, Reddy K, Chorneyko K, et al. Primary small cell carcinoma of the ureter. Canadian J Urol 2005; 12 2603-6.

3. Khattar NH, Lele SM, Kaetzel CS. Down-regulation of the polymeric immunoglobulin receptor in non-small cell lung carcinoma: correlation with dysregulated expression of the transcription factors USF and AP2. J Biomed Sci 2005; 12: 65-77.

4. Nakamura Y, Tajiri T, Uchida E, et al. Changes to levels of serum neuron-specific enolase in a patient with small cell carcinoma of the pancreas. J Hepatobiliary Pancreat Surg 2005; 12: 93-8.

5. Rebelatto MC, Midha A, Mistry A, et al. Development of a programmed cell death ligand-1 immunohistochemical assay validated for analysis of non-small cell lung cancer and head and neck squamous cell carcinoma. Diagnostic Pathol 2016; 11: 95.

6. Esteller M. Non-coding RNAs in human disease. Nat Rev Genet 2011; 12: 861-74.

7. Zhu Z, Wang G, Ma K, et al. GABAergic neurons in nucleus accumbens are correlated to resilience and vulnerability to chronic stress for major depression. Oncotarget 2017; 8: 35933-45.

8. Ma K, Xu A, Cui S, et al. Impaired GABA synthesis, uptake and release are associated with depression-like behaviors induced by chronic mild stress. Transl Psychiatry 2016; 6: e910.

9. Ma K, Guo L, Xu A, et al. Molecular mechanism for stress-induced depression assessed by sequencing miRNA and mRNA in medial prefrontal cortex. PloS One 2016; 11: e0159093.

10. Eder P, Lykowska-Szuber L, Krela-Kazmierczak I, et al. Disturbances in apoptosis of lamina propria lymphocytes in Crohn's disease. Arch Med Sci 2015; 11: 1279-85.

11. Wang GC, He QY, Tong DK, et al. MiR-367 negatively regulates apoptosis induced by adriamycin in osteosarcoma cells by targeting KLF4. J Bone Oncol 2016; 5: 51-6.

12. Chen X, Chen XG, Hu X, et al. MiR-34a and miR-203 inhibit survivin expression to control cell proliferation and survival in human osteosarcoma cells. J Cancer 2016; 7: 1057-65.
13. Li RZ, Wang LM. Decreased microRNA-452 expression and its prognostic significance in human osteosarcoma. World J Surg Oncol 2016; 14: 150.

14. Liu $M$, Xiusheng $H$, Xiao X, et al. Overexpression of miR$422 a$ inhibits cell proliferation and invasion, and enhances chemosensitivity in osteosarcoma cells. Oncol Rep 2016; 36: 3371-8.

15. Hori D, Dunkerly-Eyring B, Nomura Y, et al. miR-181b regulates vascular stiffness age dependently in part by regulating TGF-beta signaling. PloS One 2017; 12: e0174108.

16. Zheng Y, Lv X, Wang X, et al. MiR-181b promotes chemoresistance in breast cancer by regulating Bim expression. Oncol Rep 2016; 35: 683-90.

17. Tian F, Shen Y, Chen Z, et al. Aberrant miR-181b-5p and miR-486-5p expression in serum and tissue of nonsmall cell lung cancer. Gene 2016; 591: 338-43.

18. An TH, He QW, Xia YP, et al. MiR-181b antagonizes atherosclerotic plaque vulnerability through modulating macrophage polarization by directly targeting Notch1. Mol Neurobiol 2016 (PMID:27722924).

19. Yu F, Zhou G, Li G, et al. Serum miR-181b is correlated with hepatitis $B$ virus replication and disease progression in chronic hepatitis B patients. Dig Dis Sci 2015; 60: 2346-52.

20. Peng Z, Li J, Li Y, et al. Downregulation of miR-181b in mouse brain following ischemic stroke induces neuroprotection against ischemic injury through targeting heat shock protein $\mathrm{A} 5$ and ubiquitin carboxyl-terminal hydrolase isozyme L1. J Neurosci Res 2013; 91: 1349-62.

21. Tao T, Wang Y, Luo H, et al. Involvement of FOS-mediated $\mathrm{miR}-181 \mathrm{~b} / \mathrm{miR}-21$ signalling in the progression of malignant gliomas. Eur J Cancer 2013; 49: 3055-63.

22. Wang J, Sai K, Chen FR, et al. miR-181b modulates glioma cell sensitivity to temozolomide by targeting MEK1. Cancer Chemother Pharmacol 2013; 72: 147-58.

23. Guo JX, Tao QS, Lou PR, et al. miR-181b as a potential molecular target for anticancer therapy of gastric neoplasms. Asian Pac J Cancer Prev 2012; 13: 2263-7.

24. de Yebenes VG, Belver L, Pisano DG, et al. miR-181b negatively regulates activation-induced cytidine deaminase in B cells. J Exp Med 2008; 205: 2199-206.

25. Yang L, Wang YL, Liu S, et al. miR-181b promotes cell proliferation and reduces apoptosis by repressing the expression of adenylyl cyclase 9 (AC9) in cervical cancer cells. FEBS Letters 2014; 588: 124-30.

26. Wang B, Li W, Guo K, et al. miR-181b promotes hepatic stellate cells proliferation by targeting p27 and is elevated in the serum of cirrhosis patients. Biochem Biophys Res Commun 2012; 421: 4-8.

27. Wang X, Chen X, Meng Q, et al. MiR-181b regulates cisplatin chemosensitivity and metastasis by targeting TGFbetaR1/Smad signaling pathway in NSCLC. Sci Rep 2015; 5: 17618

28. Sun YC, Wang J, Guo CC, et al. MiR-181b sensitizes glioma cells to teniposide by targeting MDM2. BMC Cancer 2014; 14: 611.

29. Li P, Lu X, Wang Y, et al. MiR-181b suppresses proliferation of and reduces chemoresistance to temozolomide in U87 glioma stem cells. J Biomed Res 2010; 24: 436-43.

30. Lyu Z, Mao Z, Wang H, et al. MiR-181b targets Six2 and inhibits the proliferation of metanephric mesenchymal cells in vitro. Biochem Biophys Res Commun 2013; 440: 495-501.

31. Li H, Hui L, Xu W. miR-181a sensitizes a multidrug-resistant leukemia cell line K562/A02 to daunorubicin by targeting BCL-2. Acta Biochim Biophys Sinica 2012; 44: 269-77. 
32. Wang XF, Shi ZM, Wang XR, et al. MiR-181d acts as a tumor suppressor in glioma by targeting K-ras and Bcl-2. J Cancer Res Clin Oncol 2012; 138: 573-84.

33. Cittelly DM, Das PM, Salvo VA, et al. Oncogenic HER2\{Delta\}16 suppresses miR-15a/16 and deregulates $\mathrm{BCL}-2$ to promote endocrine resistance of breast tumors. Carcinogenesis 2010; 31: 2049-57.

34. Yang J, Cao Y, Sun J, et al. Curcumin reduces the expression of Bcl-2 by upregulating miR-15a and miR-16 in MCF-7 cells. Med Oncol 2010; 27: 1114-8. 